\title{
Intelligent Assistant System for the Automatic Assessment of Fall Processes in Sports Climbing for Injury Prevention based on Inertial Sensor Data
}

\begin{abstract}
In this work, an assistant system is presented for the automatic assessment of falling and belaying in sport climbing. Both climber and belayer are equipped with inertial measurement unit (IMU) sensors. Forces as well as movements in the form of multi-dimensional accelerations on the legs and torso are captured. It can be shown that forces can be estimated by means of IMU sensors, thus eliminating a complex force measurement unit in the safety chain. Furthermore, the data can be used to assess both falling and belaying by automatic segmentation and evaluation algorithms. The sensor data should later be evaluated automatically in order to objectively measure faulty behavior by climber or belayer (for example wrong jump-off behavior, too hard protection, etc.). The overall goal is to provide quantified feedback in fall training for injury and accident prevention.
\end{abstract}

Keywords: Accident prevention, sports climbing, fall analysis, wireless body sensor networks, inertial measurement units

https://doi.org/10.1515/cdbme-2019-0047

\section{Introduction}

In recent years, sports climbing gained enormous popularity and is practiced not only on rock but also in climbing gyms. A climbing rope is used for belaying the climber, which is attached to multiple anchor in the wall using quickdraws (a combination of slings and carabiners). However, studies show a large number of safety failures, which are the main cause of accidents in climbing gyms $[1,2]$. Many injuries are most likely caused by overtightening, e.g. hard belaying, resulting into strong impacts into the wall [3]. The German Alpine Club DAV study about

\footnotetext{
*Corresponding author: Michael Munz: University of Applied Sciences Ulm, Germany, Michael.munz@thu.de Thomas Engleder: University of Applied Sciences Ulm, Germany O Open Access. @ 2019 Michael Munz et al., published by De Gruyter. (c) BY NoDerivatives 4.0 License.
}

climbing gym in 2012 recorded a total of 161 accidents involving serious injuries (ambulance use) in 31 climbing gyms. In this study, the behaviour during belaying was also examined and an average of 1.4 belaying errors per lead climb and 0.7 errors per top-rope [1] were recorded. However, this doesn't mean that every belay error automatically leads to an accident. Usually a combination of errors and unfortunate circumstances is necessary, such as the release of the rope with simultaneous fall of the climber, a too long jump off the fall and subsequent striking on a volume, etc. Faulty belaying thus rarely leads to an accident and therefore nearly no feedback to the belayer is provided. This can lead to a dangerous adaptation. One possibility to realize injury prevention is specialized fall training, which individually improves the technique and reduces the frequency of mistakes. However, mistakes in belaying are independent of climbing ability: the climbing experience or climbing difficulty does not correlate with the quality of belaying. Therefore, even experienced climbers are not protected from belay errors. Fall and safety training should regularly be practiced not only by beginners, but also by experienced climbers. The basic idea of the fall and safety instructor presented in this work provides a starting point for training evaluation and objective feedback of the quality to both climber and belayer. For this, both belayer and climber are equipped with inertial measurement unit (IMU) sensors. The transmission of the measured data is done with Bluetooth, the evaluation is possible on a notebook, tablet or even smartphone. The system can be used universally in every route and with every backup device. Training feedback can be used to monitor continuous improvement. A similar sensor system was presented in [4], but only climbing falls were detected, no further assessment of quality aspects was provided.

One of the most important factors of a good belaying technique is the distinguishing between dynamic and hard belaying. Soft belaying is achieved when the belayer moves towards the wall upon breaking. Hard belaying is achieved when the belayer either stays passive in his position or even moves away from the wall. Although prolongating the fall 
length, dynamic belaying reduces the maximum deceleration force. Hard belaying introduces additional force into the system, resulting in a strong impact to the climber towards the wall, which often leads to injuries of the climber. Therefore, the dynamic of the belay technique is important to prevent injuries [5].

The quantities to be acquired by the system are:

- Forces on the climber

- Fall duration, fall distance

- Accelerations on the legs of the climber during impact

- Trajectory of the climber during fall (rotation, etc.)

- Movement of the belayer (upwards, downwards, etc.)

Sensors can determine these quantities metrologically. From the measured data, quality criteria (scores) for the fall and the belay process are to be calculated and evaluated in a next step. These are among others:

- Strength of the impact, dynamics of belaying

- Adequacy of the amount of slack rope

- Jumping behaviour of the climber (too far away from the wall, rotation, etc.)

- Impact on the wall and force reduction

- Stance position of the belayer

- Reaction of the belayer (passive / active, etc.)

The scores should provide an objective measure of the quality of the fall course, broken down into climbers and belayer aspects.

\section{Material and Methods}

\subsection{Measurement principle}

Measurements include force, acceleration, orientation and short-term position, and their temporal change. To detect forces usually load cells are used, which are inserted into the safety chain. However, this introduces an additional risk (breakage, wrong integration, etc.) and affects belayer and climber in their normal behaviour. Therefore, in this work we show that forces can be reconstructed from the IMU data. To validate this method, a load cell with a measuring range of \pm $10 \mathrm{kN}$ was used.

To measure the movement and accelerations, IMU sensors (MPU-9150, InvenSense) were attached to climber and belayer. Each IMU consists of three-axis acceleration, gyro and magnetic field sensors. All data is digitally recorded by a microcontroller and transmitted wirelessly via Bluetooth to a computer, allowing synchronous data acquisition of several IMUs. The measurement rate was $100 \mathrm{~Hz}$.

\subsection{Execution of Trials}

Several trials were executed in order to prove the basic feasibility of metrological acquisition of the required quantities by means of IMUs. Forces are evaluated by means of force measuring sensors. However, this should later be completely replaced by IMU measurements.

In total, 16 trials with different conditions were carried out in a climbing gym during toprope and lead falls (see table 1). In toprope, some of the falls were deliberately soft or very dynamic, some passive or even with hard belay. The fall lengths in toprope situation were about $2 \mathrm{~m}$ each. This corresponds to the amount of slack rope deliberately issued by the belayer. In the lead, the last quickdraw was exceeded by $0.7 \mathrm{~m}$ to $1.5 \mathrm{~m}$, resulting in fall lengths of $1.5 \mathrm{~m}$ to $3 \mathrm{~m}$.

IMU sensors were attached to the body's centre of gravity (COG) of both climber and belayer and to the climber's ankles. The attachment of the sensor centrally on the back of the waist belt is convenient to map the attack of the gravitational force at the centre of mass. The acceleration sensors on the ankles should provide additional information about the impact energy when contacting the wall. In addition, the load cell was inserted into the safety chain at the climber's central belay loop at the harness. In this paper, we only present climber's data.

In a climbing gym, DIN 2015 / 2015-07-00 defines the maximum distance between two quickdraws with height $>$ $8 \mathrm{~m}$ above ground to $1.50 \mathrm{~m}$. The trial conditions in table 1 therefore represent the maximum expected fall height in climbing without unnecessary slack rope in the system.

Table 1: List of trial conditions

\begin{tabular}{|c|c|c|c|c|}
\hline No & Type & $\begin{array}{l}\text { Free rope } \\
\text { length [m] }\end{array}$ & $\begin{array}{l}\text { Slack } \\
\text { rope } \\
{[\mathrm{m}]} \\
\end{array}$ & $\begin{array}{l}\text { Height above } \\
\text { last quickdraw } \\
\text { [m] }\end{array}$ \\
\hline 1 & \multirow{2}{*}{$\begin{array}{l}\text { Dynamic } \\
\text { belay }\end{array}$} & 15,0 & 2,0 & - \\
\hline 2 & & 15,0 & 2,0 & - \\
\hline 3 & \multirow[t]{2}{*}{ Hard belay } & 15,0 & 2,0 & - \\
\hline 4 & & 15,0 & 2,0 & - \\
\hline 5 & \multirow[t]{2}{*}{ Wide jump } & 15,0 & 2,0 & - \\
\hline 6 & & 15,0 & 2,0 & - \\
\hline 7 & \multirow{3}{*}{$\begin{array}{l}\text { "Normal" } \\
\text { conditions }\end{array}$} & 12,0 & 2,0 & 1,0 \\
\hline 8 & & 12,0 & - & 1,0 \\
\hline 9 & & 12,0 & - & 1,0 \\
\hline 10 & \multirow{7}{*}{$\begin{array}{l}\text { Leading } \\
\text { climb with } \\
\text { different } \\
\text { conditions }\end{array}$} & 6,5 & - & 0,7 \\
\hline 11 & & 8,0 & - & 0,7 \\
\hline 12 & & 9,0 & - & 1,5 \\
\hline 13 & & 10,0 & - & 0,7 \\
\hline 14 & & 11,0 & - & 1,5 \\
\hline 15 & & 11,0 & - & 0,7 \\
\hline 16 & & 12,0 & - & 1,5 \\
\hline
\end{tabular}


based on Inertial Sensor Data - 185

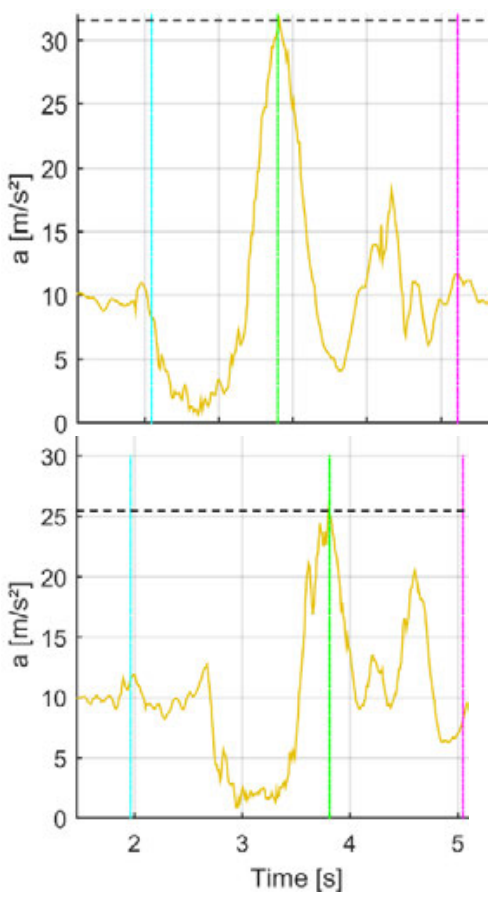

Figure 1: Resulting acceleration (yellow) and segmented phases / events during fall: jump start (blue), jump end (magenta), max. acceleration (green) measured at the COG of the climber. Top: hard belay, bottom: dynamic

\subsection{Data processing}

From the data of the inertial sensors it is possible to deduce the orientation of the sensor at any time. For this, a gradient decent algorithm [6] for fusing acceleration and gyro measurements of the IMU sensors is used. In addition to the accelerations, this also allows the body movements and body position to be determined, which provide essential information for the later scores.

The evaluation of the measurement results focuses primarily on the measurement data of the force sensor and the acceleration values at the COG. Supplementary information is obtained by the determined acceleration values at the ankles. The coordinate axes of the acceleration sensors span a Cartesian coordinate system. The resulting absolute acceleration $a_{\text {Reg }}$ can be calculated based on the accelerations along the $\mathrm{x}$-, $\mathrm{y}$ - and $\mathrm{z}$-axis using $a_{\mathrm{RES}}=\sqrt{a_{x}{ }^{2}+a_{y}{ }^{2}+a_{z}{ }^{2}}$

Based on $a_{\text {REg }}$, the fall can be segmented into different phases, as shown in figure 1. Start and end of the fall, time and strength of the maximum impact into the rope are automatically calculated by a segmentation algorithm. For this purpose, various filters and pattern recognition techniques are applied to the measurement data.

First, the data of the acceleration sensor is low pass filtered for noise reduction. Afterwards, the impulse is estimated from the acceleration data using $p=m \int a$, where the integration is realized using a numerical integration method. The initial impulse of the fall is fit using linear regression, which corresponds to an unrestrained fall. The greatest deviation of the impulse from the regression line is at impact into the rope, which leads to the braking timestamp. Subsequently, the beginning and end of the fall are detected. For this purpose, a resting position is detected for the end of the fall, when only gravitational acceleration is measured for a certain period of time (after the decay phase). The start of the free fall is detected by comparing $a_{\text {Res }}$ to a low threshold (additional acceleration due to the rope friction and the redundant belay needed for the trials). By this, the duration of the free fall is defined. The fall length can be calculated by double-integrating the acceleration. This is applicable because of the short integration time and the low acceleration noise.

With the known body weight $m$ of the climber, the force at the central belay loop of the climber can be estimated from the resulting acceleration using $F_{B}=m \cdot a_{\text {Res }}$.

The timestamps of the maxima during the deceleration process (impact force) in both $F_{B}$ and measured force $F$ are determined. The time offset and the difference between the maximum values serve as a measure of the correspondence between the estimated value $F_{B}$ and the real force measurement $F$.

\section{Results and discussion}

The evaluation of the force measurements shows a temporal deviation of $0.01 \mathrm{~s}$ (median) with a range of -0.04 to $+0.03 \mathrm{~s}$ (1st or 3rd quartile) over all measurements. The duration of the impact peak according to [7] is between $0.1 \mathrm{~s}$ and $0.2 \mathrm{~s}$. Therefore, the results are within the time window of the impact peak, and so the maximum impact was always detected correctly.

For the deviation of the estimated force $F_{B}$ from the measured force $F$, the following values were obtained for all experiments: Median: $-112 \mathrm{~N}$, range: $-512 \mathrm{~N}$ to $-102 \mathrm{~N}$ (1st and 3rd quartile, respectively). In addition to the force peaks, the course of the estimated and measured force was also examined. For this purpose, the course from the beginning of the deceleration process to the capture impact was subjected to a congruence analysis. Figure 2 shows results of the individual trials as box plots. All values are normalized to the maximum observed force of $2050 \mathrm{~N}$.

The difference during the phase of the free fall is due to the rope friction in the carabiners of the quickdraws, leading to greater differences in trials with longer falls.

Analysing the acceleration data in more detail, additional assessment can be conducted. The strength of the impact 


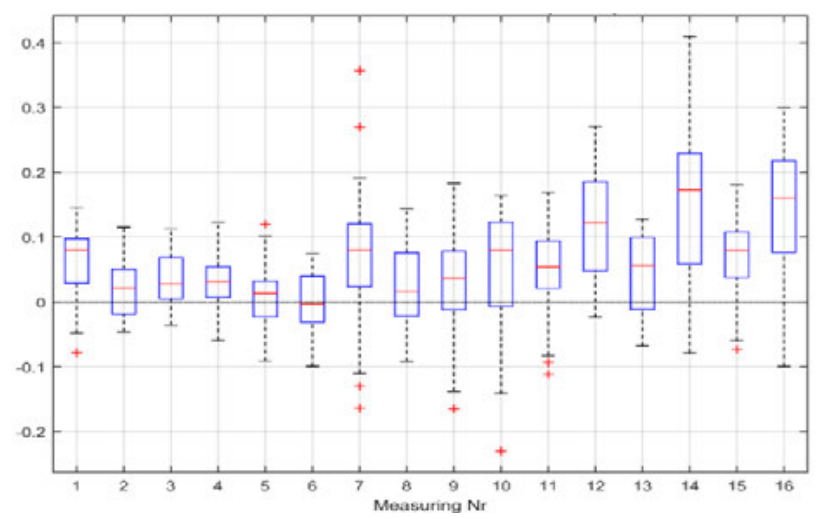

Figure 2: Boxplot of the difference between measured force $\mathrm{F}$ and FB for all 16 experiments. The deviation between $F$ and FB normalized to $2050 \mathrm{~N}$ is indicated on the y axis.

peaks allows the differentiation between soft and hard belaying. Unnecessarily strong initial take-off of the climber can also be detected using acceleration data and the direction of acceleration. By measuring the accelerations on the ankles, the delay between impact of both feet on the wall can be detected. This serves as an indication of an unstable posture and thus a poor control of the fall. Figure 3 shows a corresponding example: after the impact and breaking start in the rope, the feet touch the wall asynchronously (two peaks). In addition, the strength of the impact of feet on the wall can be determined. The shape of this peak can also serve as a feature for assessment of the impact attenuation by the climber's feet.

\section{Conclusion and Outlook}

In this work, a prototype of a fall and safety instructor for the quantitative assessment of forces and movements in sport climbing falls is presented, providing objective feedback to the athletes or coach. To the best of the authors' knowledge, this is the very first system that can perform such an assessment in sports climbing for injury prevention.

Validation measurements show that the impact force at the climber's rope point can be estimated using IMU measurement data, allowing to quantify the softness of the belaying technique, as one important factor for injury prevention. With a first analytical evaluation of the IMU sensor data, we showed that it is possible to differentiate various processes and effects, allowing assessment of both belay and fall quality.

A frequently discussed, very important parameter that cannot yet be measured and evaluated with the current system

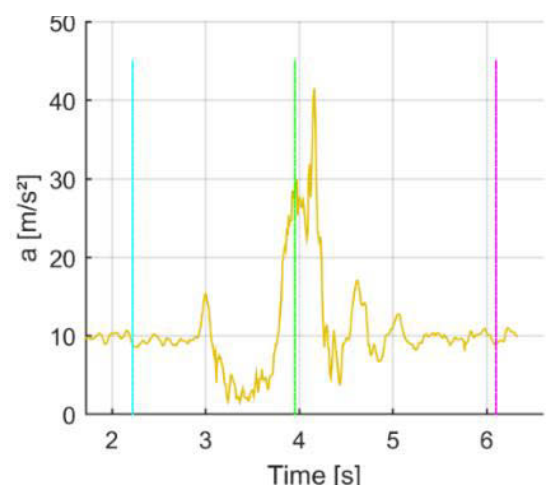

Figure 3: Example of a fall with asynchronous impact of feet after the main deceleration peak of the rope impact.

is the amount of slack in a fall. This aspect must be assessed in relation to the climbing height. Sensory extensions are planned, which will be evaluated in subsequent work, allowing an evaluation of a possible fall situation at any time. Further work has been done in developing algorithms for scoring relevant quality criteria for safer and climbers on the presented features extracted from acceleration data. The output of this scoring algorithm can then be evaluated as part of a series of measurements with independent climbing coaches.

\section{Author Statement}

Research funding: The authors state no funding involved. Conflict of interest: Authors state no conflict of interest.

\section{References}

[1] Hummel, C. \& Hellberg, F. „Unfälle an künstlichen Kletterhallen: Kampf dem Eisberg!“. DAV Panorama: Magazin des Deutschen Alpenvereins 6/2014, 62-65.

[2] Schöffl, V.R., Hoffmann, G. \& Küpper, T. (2013): Acute injury risk and severity in indoor climbing-a prospective analysis of 515,337 indoor climbing wall visits in 5 years. - Wilderness \& environmental medicine 24, 3, 187-194.

[3] Radelzhofer, P. (2014500): Bergunfallstatistik 2012-2013. München.

[4] Tonoli (2011): Fall identification in rock climbing using wearable device. In: Journal of Animal Science (X), S. 1-11. DOI: 10.1177/ToBeAssigned.

[5] Schöffl, Volker; Morrison, Audry; Schöffl, Isabelle; Küpper, Thomas (2012): The epidemiology of injury in mountaineering, rock and ice climbing. In: Medicine and sport science 58, S. $17-$ 43. DOI: $10.1159 / 000338575$.

[6] Madgwick, S. O., Harrison, A. J., \& Vaidyanathan, R. (2011): "Estimation of IMU and MARG orientation using a gradient descent algorithm". In Rehabilitation Robotics (ICORR), 2011 IEEE International Conference on (pp. 1-7). IEEE.

[7] Fimml, W. \& Larcher, M. (2000): „Energie ist Kraft mal Weg: Sicherungstheoretische Grundlagen, Teil 2“. bergundsteigen: Zeitschrift für Risikomanagement im Bergsport 4/00, 14-20. 